\title{
Research on the state of social media studies in Malaysia: 2004-2015
}

\begin{abstract}
In the recent years, the advancement of social media plays a phenomenal role in the Malaysia's media and academic landscape. Social media have garnered massive adoption among the online population, paving way for scholars to understand on the usage and impact of social media on the users in the country. The rising amount of research on social media illustrates a growing interest in this phenomenon. Despite the budding interest among scholars, the understanding on the research patterns and status on social media studies in Malaysia is still inadequate, comparing with the research trends analyses in the West. This paper investigated the trends of social media research in Malaysia from 2004 to 2015. A quantitative content analysis was conducted on 79 articles in 21 selected academic journals in identifying the methodological approach, theoretical application, topical, social media type, type of content and research developmental phases of social media scholarship. Findings revealed social media research in Malaysia is still in formative stage but increasing throughout the years. Unlike findings from past analyses, theory-driven articles in Malaysia were more prevalent than theoretical presence in the West. Nonetheless, Malaysian scholars still lack in conceptualisation and expansion of theories. The study suggests for a more explicit theoretical foundation and technology advancement in the future research. This study also suggests that future scholarly endeavours should have better methodological rigour and wider dispersion of social media medium and content type.
\end{abstract}

Keyword: Social media; Research trends; Content analysis; Malaysian users; Scholarly work 